\title{
A Biomimetic Study of Discontinuous-Constraint Metamorphic Mechanism for Gecko-Like Robot
}

\author{
ZhenDong Dai and HongKai Li \\ Nanjing University of Aeronautics and Astronautics
}

China

\section{Introduction}

Mobile robots have been strong demands for defense, surveillance and counter terrorism missions as a moving platform under unconstrained environments (Eric \& John, 1999; Robert et al., 2003). Two moving mechanisms, wheeled and legged, were used for mobile robots. Both of them have been studied for years. Wheeled vehicles could move much faster and have much higher efficiency than that of legged ones (Todd, 1985); they are widely used in well-structured circumstances, such as automobile and train has to be move on highway or railroad. The key point of wheeled motion is that the wheels continuously contact to the substrate. This demand makes the wheeled vehicles moving on flat surface under tens to hundreds times higher efficiency than that of legged locomotion (Todd, 1985).

On the other hand, a lot of animals that live on land have selected the legged mechanism for their locomotion. This selection comes from the advantages of legged mechanism, highly adaptability to the unstructured natural circumstance, multifunctional behaviours, such as walking, running, jumping and even climbing, and strong function compensation. The advantages that make the animals to live much more reliably were high energy efficient, and what's important is highly adaptive, robust and reliable for locomotion. Those characters are also needed for the moving system under unconstructive circumstance, unmanned intelligent situation, such as the area of defence, surveillance and counter terrorism missions, where no road existed. The legged locomotion mechanism has been demonstrated successfully in wild bumpy circumstance, so mimicking the motion mechanism and control pattern of the legged animals may greatly increase the locomotion abilities, though it's difficult to copy nature (Siegwart \& Nourbakhsh, 2004).

Biomimetics on locomotion aims to reveal the secrets of legged locomotion of animals in order to understand how animals' motion and to get inspiration by the technique animals used. Now a lot of robots inspired by animals were developed in laboratory, but their performance of locomotion, such as walking, jumping, running, sliding, swimming, lagged far behind their natural counterparts in stability, agility, robustness, environmental adaptability and energy efficiency (Dickinson et al., 2000). Humanoid robots could play the 
piano, dance and play Chinese GongFu, but they could not work for more than 30 minutes because of the energy supply. Research showed that the best humanoid robot, ASIMO, consumed ten times energy of human for the same motion (Collins et al., 2005). The energy efficiency of the best snake-like robot was about $25 \%$, but a snake can move faster and longer even without food for several weeks. What makes such a big difference in energy efficiency between robots and its animal prototypes?

After millenniums years of evolution, a lot of animals that live on earth have optimized the legged mechanism for locomotion and feet for stable contact with the substrate. They selected flat hoofs with various toes to reduce the contact stress and developed several joints between bones of the foot to adapt the slope of the substrate. Research on legged machines could lead to the construction of useful legged vehicles and help us to understand legged locomotion in animals (Raibert, 1986). Developments of RHex were inspired by the biomechanics of arthropod locomotion (Altendorfer et al., 2001). The outstanding mobility of Bigdog results from the study on locomotion of dog (Raibert et al., 2008).

Gecko, have been a study highlights because of their excellent climbing ability, is a perfect object for wall-climbing robot. As a scansorial quadruped animal, gecko's locomotion abilities benefit from the adhesive pads, short strong limbs and flatten body (Cartmill, 1985; Damme et al., 1997). Morphology , kinematics (Damme et al., 1998; Damme et al., 1997; Li et al., 2009; Zaaf et al., 2001) and dynamics (Autumn et al., 2006) related to gecko's locomotion have been extensively studied. The adhesive mechanism was revealed (Autumn et al., 2000) as van der Waals force between the hierarchical structural setae on toes and substrate. Inspired by the geometric structure of seta, Carbon nanotube arrays was developed (Qu et al., 2008), the array showed strong shear binding-on and easy normal lifting-off character and may be wonderful artificial adhesive pads for gecko-like robot. Other adhesive pads were developed (Santos et al., 2007) and successfully used in a climbing robot-Sticybot (Kim et al., 2008). Though there are a lot of researches on gecko, the mechanism theory and related controlling technique are still unclearly.

In this paper, the mechanism of a gecko-like sprawling robot was proposed in section 2. In section 3 we analyzed the kinematic and dynamic characters of the robot mechanism. For well understanding how gecko moves we observed the angular regularity of gecko's each joints when moving on horizontal and vertical surface in section 4 . Motion scheme including walking gait and trotting gait was introduced in section 5. And in section 6 we designed a realizable control system.

\section{Mechanism of legged gecko-like robot}

Limbs were the executive machine of geckos that consisted of skeleton-joint-muscle. Skeleton moved relied on the rotation of joints, and carry foot to the next support position or drive body forward. A legged mechanism for gecko-like robot (Fig. 1 A) (Dai et al.) was proposed according to the anatomic studies on gecko (Liu et al., 2005; Zaaf et al., 1999), which was generally considered as a multi-jointed serial chain (Winters \& Crago, 2000). One step cycle includes an swing phase and a stance phase. During swing phase the leg lifts off and forms an open chain (Fig. 1 B). But in stance phase two or three feet, the terminal 
part of legs, stably contact on the substrate and propel the body move forward, which form a closed chain by two limbs (or some time three limbs) (Fig. 1 C). When limbs of gecko shift from an swing phase to stance phase, the constrained forces acted on the foot changes discontinuously, the mechanism of the locomotion changes because of the shift from open chain to closed chain and the change of length of the equivalent bar (frame) in each leg step. We define this phenomenon as discontinuous constraint metamorphic linkage mechanism (DC-MLM). The metamorphic mechanism come from the definition given by Dai (Dai \& Jones, 1999). The degree of freedom of (DoF) the cardan joint $J_{\mathrm{BF}}(J$ represents Joint, subscript letters BF represents Body and Femur), the revolute joint $J_{\mathrm{FT}}$ (FT represents Femur and Tibia), and the spherical joint $J_{\mathrm{TD}}$ (TD represents Tibia and Dactylust) are 2, 1, 3 respectively.
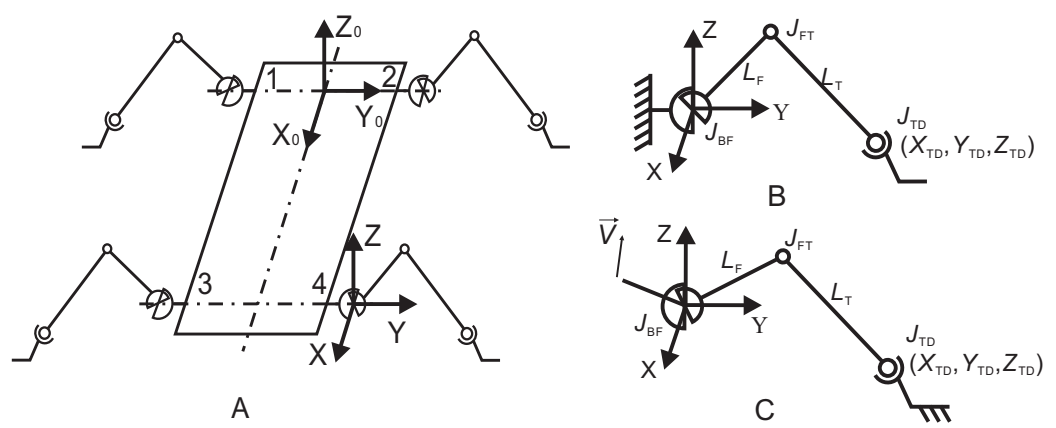

Fig. 1. A simplified mechanism of gecko-like robot (A); swing phase (B) and stance phase (C) of one leg. The gecko-like robot composed of four legs, on each leg there are two active joints $J_{\mathrm{BF}} \& J_{\mathrm{FT}}$ and one passive joint $J_{\mathrm{TD}}$.

The adhesive force would increase with increase of real contact area, and the feet of limbs under stance phase must contact with the substrate on enough "real" area, so joint $J_{\mathrm{TD}}$ has evolved as a spherical joint to adapt the sole with the target surface. The mechanism needs to be driven by three active independent actuators at least (the $J_{\mathrm{TD}}$ as a passive joint) to allow the foot of the robot to reach the intended position and to retain enough mobility when the limb is at swing phase. The similar change was performed in folding carton, on which the metamorphic mechanism was proposed and developed (Dai \& Jones, 1999; Jin et al., 2004; Jin et al., 2005).

In order to drive the robot synchronously, glenohumeral joint or Joint $J_{B F}$ of the legs at stance phase must have the same speed vector, which need actuators of each leg drive on a concordant way. How to make them driven concordant is still a big problem. Right now spherical shaped foot was selected by a lot of legged robots that move on ground, because the design increases the adaptability of foot with substrate without passive joint. On the other hand, this foot could not meet the requirement of gecko-like robot where enough real contact area is needed to generate enough adhesive force.

There are three contact status between foot of a gecko-robot and the substrate-non-contact, sliding contact and stable contact, which is presented by the interaction force $F_{S}-$ friction force parallel to the target surface and adhesive or repulsive force perpendicular to the 
surface as following:

$$
\left\{\begin{array}{lll}
F s=0 & \text { open chain } & \\
F s=C & \text { close loop chain } & \text { Slide } \\
\text { Fs }=\infty & \text { close loop chain } & \text { Stable }
\end{array}\right.
$$

For the first case, the foot was at swing phase and lift off the substrate, so Fs would be zero, the methods of analyzing the mechanism, kinematics and dynamics of an open-chain are well developed. When the leg is at stance phase, foot contacted with substrate in two ways. When foot stably contacted on substrate, which generally falls in a self-locking situation, the contact force would increase with increase of the propel force. When foot slides on the surface, interaction force between foot and substrate would be determined by the frictional coefficient and normal force and the length of links (bar) of frame would change with sliding. This situation is much more complicated and will not discuss here.

When a legged mechanism moves, the power needed for motion should be discussed according two cases. When legs are in swing phase, the power needed is to overcome the force of inertia. When the legs are under stance phase, the power needed is to support the locomotion and depended on the carried load and the related motion. To reduce the power consummation, the legs under stance phase must move on a coordinate velocity. When a leg mechanism moves in a structured circumstance, the coordination could be transferred into a geometric question. In an unstructured circumstance, the coordination greatly depends on the unpredicted next contact position; new technique has to be introduced to meet the requirement. To obtain an idea from the animals' locomotion, it was necessary to understand the kinematics, dynamics and the control strategies of animals.

\section{Kinematic and dynamic analysis}

When a leg is under swing phase, it is a serial open chain with 3 active degrees of freedom with one passive sphere joint at the terminal of the leg. From the kinematics of the open chain, we will obtain the information about the working space and the trajectory of the mechanism. So the coordinates $J_{\mathrm{TD}}\left(X_{\mathrm{TD}}, Y_{\mathrm{TD}}, Z_{\mathrm{TD}}\right)$ of foot point (end point of shank) in the base frame $\Sigma-X Y Z$, which was coincident with $\mathrm{OX}_{0} \mathrm{Y}_{0} \mathrm{Z}_{0}$, could be obtained by forward kinematics as following (see Fig. $1 \mathrm{~B}$ ), Where $L_{\mathrm{F}}$ and $L_{\mathrm{T}}$ are the lengths of upper leg and shank, respectively, a (angle between YOZ plane and upper leg $L_{\mathrm{F}}$ ) and $\beta$ (angle between XOY plane and upper leg $L_{\mathrm{F}}$ ) are the angles between the leg and body respectively, and $Y$ represents the angle between the upper leg $L_{\mathrm{F}}$ and shank $L_{\mathrm{T}}$ :

$$
\left\{\begin{array}{l}
\mathrm{X}_{\mathrm{TD}}=\left[\mathrm{L}_{\mathrm{F}} \cos \alpha-\mathrm{L}_{\mathrm{T}} \cos (\alpha+\gamma)\right] \cos \beta \\
\mathrm{Y}_{\mathrm{TD}}=\left[\mathrm{L}_{\mathrm{F}} \cos \alpha-\mathrm{L}_{\mathrm{T}} \cos (\alpha+\gamma)\right] \sin \beta \\
\mathrm{Z}_{\mathrm{TD}}=\mathrm{L}_{\mathrm{F}} \sin \alpha-\mathrm{L}_{\mathrm{T}} \sin (\alpha+\gamma)
\end{array}\right.
$$

The velocity components of $J_{\text {TD }}$ could be derived from the time derivative of equations in (2). So it obvious that the linear velocity of foot tip was determined by the angular velocity of $a$, $\beta$ and $\gamma$. 
To adapt to the unstructured circumstances, point contact was selected by robots that move on land. To fit the unstructured circumstances and generate enough adhesive force to make gecko-like robot climb on walls or ceilings, a single foot with biggest contact area must be developed, and a passive spherical joint was assigned for the ankle joint (shank-foot connection) to make the foot contacted better.

\section{Angular observations of joints of Geckos moving on horizontal and vertical surfaces}

At present, the drive mechanism of a gecko robot depends mainly on micro-motors, and the planning and designing of motion are implemented based on angular orientation. Therefore, for the motion scheming of gecko robot, a more direct approach would be studying the orientation and angular changes of gecko's joints.

This section aims to reveal the relationship among the angles ( $\alpha, \beta$ and $\gamma)$ when gecko moves on floor and wall freely, and we expect the results would inspire us with a new idea for the motion plan of gecko-like robot.

\subsection{Observation system and Method}

A system to observe the three-dimensional locomotion behaviours of gecko was developed which consisted of a tunnel and a high speed camera (Mikrotron, MC1311 Germany) (Fig. 2). The tunnel was made up of a long flat marked track with two mirrors on left and right sides with an angle $135^{\circ}$ to the track, a transparent polymethy methacrylate plan which covered the top to avoid the animals falling down during experiments. The high speed camera was supported with a tripod and connected with a computer to set the frame frequency, pixels, start and stop. During the experiments the locomotion behaviour was digitally documented by the camera. The projection in two mirrors gives the lateral position of joints and, together with the real image, full spatial poses were obtained. The tunnel is wide enough to enable the geckos to move freely.

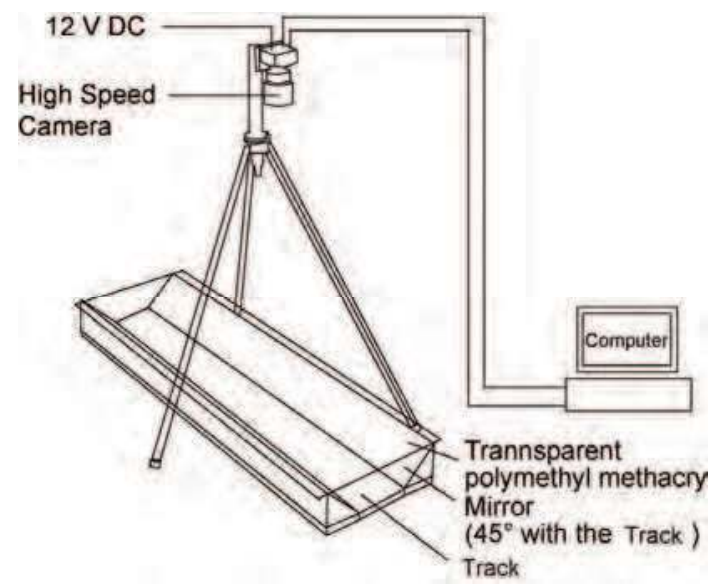

Fig. 2. Three-dimensional locomotion observation system 
The tunnel was mounted horizontally or vertically to simulate floor or wall, and the geckos were induced to move along it from one end to another.

To describe the motion clearly and be in accord with our previous work, we define the reference frame following the stereotaxic method (Wang et al., 2008). We take the underside of tunnel in the three-dimensional locomotion observation system as the horizontal plane (body plane). The sagittal plane is the plane perpendicular to the body plane and passes through the bregma and nasal points. The coronal plane is the plane through the bregma point and perpendicular to the body plane and sagittal plane. The femorotibial angle $(\alpha)$ is the angle between femur and tibia, and is always positive. The swing angle ( $\beta$ ) was calculated as the projection of the angle between femur and a plane through the coxa parallel with the coronal plane in the body plane. Swing angles in front of the parallel plane are considered positive, while behind this plane, negative. The lifting angle $(\gamma)$ is defined as the projection of the angle between femur and a plane through the coxa parallel with the body plane in the coronal plane (Fi.3). Lifting angles are considered positive before the parallel plane, and negative behind this plane. The units of all angles are in degrees $\left(^{\circ}\right)$.

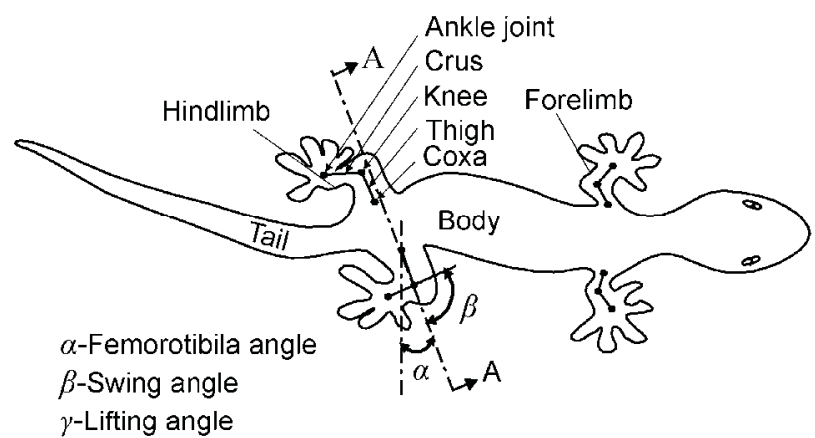

$\gamma$-Lifting angle

Fig. 3. The definition of femorotibial, swing and lifting angles

Experiments were grouped by gait speed and surface orientation, viz., the high speed trot gait (trot) and slow speed tripod gait (walk) on both horizontal and vertical surfaces. Four geckos were selected for the experiments. At least 20 trails were recorded in each experiment. Linear regression of step length against time was done to assess the steady speed, and four groups of complete sequences were selected for further analysis for which the $\mathrm{R}^{2}$ values of regression were greater than 0.95 . All geckos selected were marked with white non-toxic painted dots on the coxa, knee and ankle joints before experiments. The camera axis was perpendicularly oriented to the locomotion surface (track) and adjusted until there was a clear image in the computer (Fig. 4). The motion process was digitally recorded with a fixed frame frequency. 


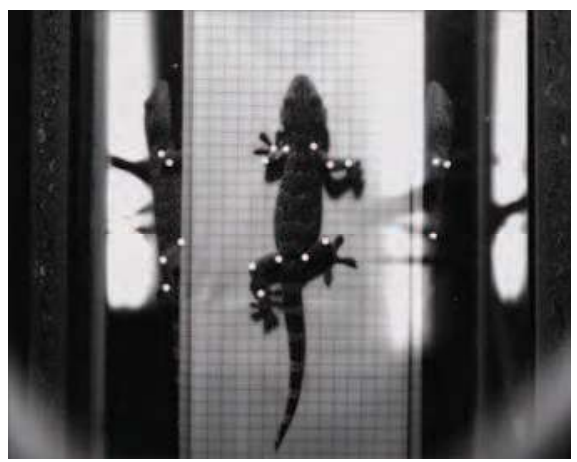

Fig. 4. One image of a free vertically climbing gecko

The middle is the real image of gecko during motion, and images on the left and right sides are the mirror images. The white plots on the images are the key points used to calculate the angles.

\subsection{Ranges of each joints}

From the experimental data, it was found that there are no speed limitations in gait alternation between trotting and walking. In other words, feet at diagonal direction might lift off and touch down at the same time even at slower velocities. Generally, when speeding up, geckos would transit from walking to trotting. The following four groups of data were selected for analysis and comparison: walking $(66.7 \mathrm{~mm} / \mathrm{s})$ and trotting $(337.1 \mathrm{~mm} / \mathrm{s})$ over a horizontal surface, and walking $(30.6 \mathrm{~mm} / \mathrm{s})$ and trotting $(241.5 \mathrm{~mm} / \mathrm{s})$ up a vertical surface. Extrema and ranges of fore and hindlimb angles are shown in Table 1.

\begin{tabular}{|c|c|c|c|c|c|c|c|c|c|}
\hline \multirow{3}{*}{ Projects } & & \multicolumn{4}{|c|}{ Forelimb } & \multicolumn{4}{|c|}{ Hindlimb } \\
\hline & & \multicolumn{2}{|c|}{ Horizontal } & \multicolumn{2}{|c|}{ Vertical } & \multicolumn{2}{|c|}{ Horizontal } & \multicolumn{2}{|c|}{ Vertical } \\
\hline & & Trot & Walk & Trot & Walk & Trot & Walk & Trot & Walk \\
\hline \multirow{3}{*}{ Swing angle $\left(^{\circ}\right)$} & Max. & 59.2 & 59.0 & 36.7 & 33.7 & 77.2 & 85.1 & 82.6 & 79.0 \\
\hline & Min. & $\square 79.2$ & $\overline{\square 72.0}$ & $\square 87.7$ & $\square 87.3$ & $\square 44.3$ & $\square 31.1$ & $\square 64.9$ & $\square 53.3$ \\
\hline & Ra. & 138.4 & 131.0 & 124.4 & 121.0 & 121.5 & 116.2 & 147.5 & 132.3 \\
\hline \multirow{3}{*}{ Lifting angle $\left(^{\circ}\right)$} & Max. & 59.6 & 50.8 & 86.3 & 84.5 & 48.7 & 21.3 & 46.9 & 35.6 \\
\hline & Min. & $\square 17.4$ & $\square 11.4$ & 15.5 & 5.4 & $\square 10.7$ & $\square 18.0$ & $\square 19.7$ & $\square 16.7$ \\
\hline & Ra. & 77.0 & 62.2 & 70.8 & 79.1 & 59.4 & 39.3 & 66.6 & 52.3 \\
\hline \multirow{3}{*}{ Femorotibial angle $\left(^{\circ}\right)$} & Max. & 138.3 & 127.2 & 109.1 & 131.8 & 135.2 & 126.7 & 151.3 & 146.9 \\
\hline & Min. & 39.3 & 47.7 & 55.2 & 56.3 & 54.4 & 78.7 & 51.1 & 47.5 \\
\hline & Ra. & 99.0 & 79.5 & 53.9 & 75.5 & 80.8 & 48.0 & 100.2 & 99.4 \\
\hline
\end{tabular}

Table 1. Extrema and ranges of fore and hind-limb angles

\subsection{Angular phase diagrams}

Angular phase diagrams are used to show the relationship and tendencies of the two groups of angles with the same time variable (Kristiaan et al., 2002). The shape of the phase diagram shows the changing trend of angles in different phases, and the position in the coordinate 
system shows the angle scope. It is very convenient to compare the same group of data in different states by their phase diagrams. The phase diagrams of swing-lifting angles and swing- femorotibial angles are shown in Fig. 5. Arrows in each figure indicate the direction of joint rotation, and swing phase is at the beginning. The time interval between two sequential plots in a diagram is the same, so the speed in each phase can be evaluated by the density of dots - the slower speed of motion the denser the points. Closed loops indicate periodicity, and the enclosed area shows the changing extent of joint angles between swing phase and stance phase.

When trotting over horizontal surfaces, swing-lifting angle plots of fore and hind limbs (Figure 5(a), (e)) and the swing-femorotibial angle plot of forelimb (Figure 5(i)) are clearly different from other plots. The density of dots is greater when swinging forward than that when swinging backward in relation to the arrow's direction.
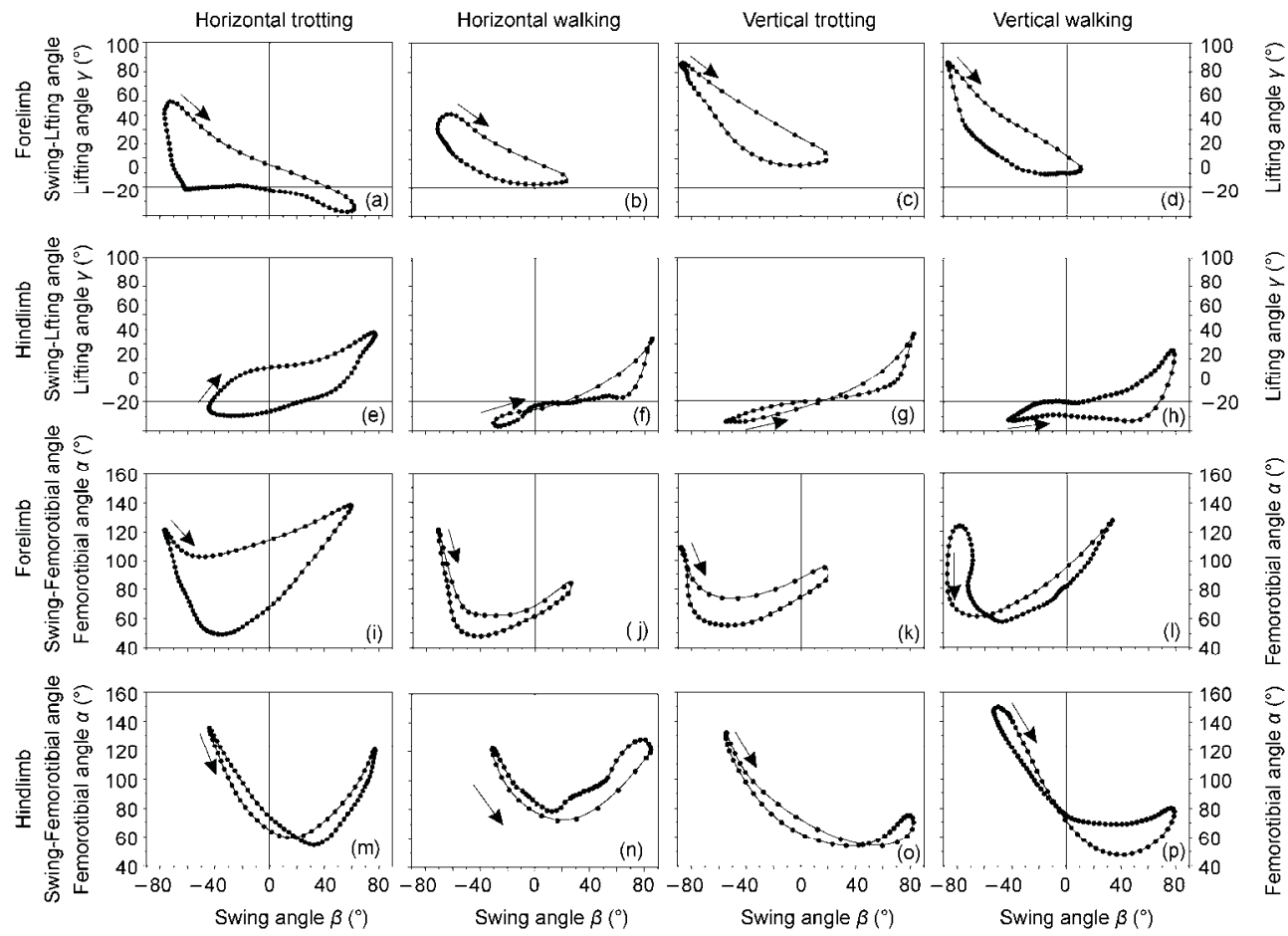

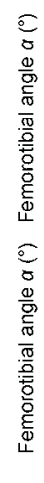

Fig. 5. Swing-lifting angle plots for forelimb trotting (a) walking; (b) on horizontal surface and trotting; (c) and walking; (c) on vertical surface; (e), (f), (g), (h) are the corresponding plots for hindlimb; (i), (j), (k), (l) are swing-femorotibial angle plots for forelimb; (m), (n), (o), (p) for Hindlimb respectively. Dots represent time slices and speed trend can be estimated by the density of dots. The arrow in each phase diagram represents the change direction of curve. Time interval of trotting and walking on horizontal surface and trotting and walking on vertical surface are $4 \mathrm{~ms}, 25 \mathrm{~ms}, 10 \mathrm{~ms}$ and $20 \mathrm{~ms}$ respectively. 
There is a great difference in the shape of phase diagrams due to the different velocities when moving over horizontal surfaces, but the swing-femorotibial angle plot of the hindlimbs are similar (Figure 5(m), (n)). The enclosed areas of the swing-femorotibial angle plot in fore and hind limbs increase with the increasing speed (Figure 5(a), (b), (e), (f)). The change of femorotibial angle in forelimb is slight in the swing phase, but large bending exists in the stance phase (Figure 5(i)). The trends of the swing-lifting angle plot for forelimb (Figure 5(a) - (d)) and hindlimb (Figure 5(e) - (h)) are reversed in correspondence with the forelimb's angle curves in Figure 4. Compared with moving on horizontal surfaces, there is a lot in common between each group of phase diagrams in different gaits up vertical surfaces. And the forelimb swing- lifting angle plots (Figure 5(c), (d)) are the most obvious among these groups. Shape almost remained the same, except the position in the coordinate system when moving up vertical surfaces, which shows that the angular velocity of joints increases with speed, but the mode of joint rotation nearly keeps invariant. The swing-lifting angle plots are higher when moving up vertical surfaces than those when moving over horizontal surfaces (Figure 5(a) - (d)), and the positions as seen in Figure 5(c), (d) are all above the zero line, which means that the thigh has swung above the plane that crosses the coxa and parallels the body plane, placed the center of the body closer to the motion surface.

\subsection{Influence of locomotion speed on joint rotation}

The trotting gait is used when geckos move at high speed, that is, the motion cycles of fore and hind legs diagonally opposed are almost the same. The triangle gait is engaged at low speeds, namely, each swing in turn is based on the order of front right - hind left - front left hind right - front right. Different strategies during speed adjustments are adopted when moving over horizontal and vertical surfaces at different gaits.

The experiment showed that stride frequency increased when speeding up, but step length, stride and duty factor remained unchanged when geckos moved over horizontal surfaces, and hindlimb moved more parasagittally. Both step length and stride frequency increased with increasing speed up vertical surfaces, but the relative increase of step length was small compared with that of stride frequency (Zaaf et al., 2001). Results joint angles exhibited the following rules: angular velocity should rise with increasing locomotion speed over horizontal surfaces, but the scope of swing angle remain unchanged, and the curve of the lifting angle in the stance phase should be lower to decrease the angle between thigh and sagittal plane; angular velocity should rise with increasing locomotion speed up vertical surfaces, and the scope of the swing angle should increase. Rotation speed of the joint should increase with increasing locomotion speed over the same surface, which could be derived from the dot density in the phase diagrams in Figure 5. The curve of the lifting angle corresponding to horizontal trotting was lower than that up vertical surfaces. Increased amplitude of hindlimb swing angle with increasing speed up vertical surfaces is obvious (Table 1). This experimental result was proved by our angular measurements. Moreover, in our experiments, the swing angle increase of the hindlimb was accomplished mainly during the backward swing, but did not change much during the forward swing.

When geckos trot horizontally, the task of the forelimb in the swing phase is completed by the forward extension of the crus carried by the forward rotation of the coxa, while the femorotibial angle almost remains unchanged at the end of the stance phase. The 
performance looks quite smart and quick. The lifting range of the fore thigh in trotting is larger than that in walking to avoid collisions between the limb and blocks (such as stones and so on) in swing phase and body drag over the locomotion surface.

Morphology differences (Zaaf et al., 1999) between forelimb and hindlimb endow the gecko with the possession of a special adaptation. The shorter forelimb is shaped to climb. The thigh moves forward at the end of the swing phase and moves mostly hindward at the end of the stance phase. The relative height between coxa and foot in the forelimb is maximal at the end of swing phase. Regarding the coordination and compliance of movement, the limbs do not move forward at the moment of lift off, but continue to move backwards to avoid the abrupt change in acceleration.

\subsection{The differences of joint rotation between moving on horizontal and vertical surfaces}

Over horizontal surfaces, the body is supported by four legs on the substratum by overcoming gravity, friction and forward propulsion. Up vertical surfaces, there is the risk of tumbling backwards because of the moment of inertia when the body's center of mass leaves the wall. This moment of inertia is proportional to the gecko's mass and the perpendicular distance between the centre of mass and the wall. The influence of the moment of inertia is compensated by the pulling force of the shorter forelimb against the wall, and the torque due to the fact that the limbs in the swing phase overcome the gravity and also offer enough propulsion to move the body upward (Autumn et al., 2006).

The moment of inertia would be decreased by reducing the distance between the centre of mass and the vertical surface. Thighs in the stance phase always above the plane that crosses the coxa and paralleling the body plane to reduce the overturning moment. The lifting angles of the hindlimb are at the lowest position during most of the motion cycle, and the curves are very flat. Locomotion velocity has little influence on each limb's motion style (the shapes of the phase diagrams remain nearly the same). Over horizontal surfaces, limbs just need to overcome gravity and support propulsion, so the motions of limbs are more sprightly and agile.

The results of locomotion observation offered us a reference to scheme the robot gaits, and make the motion smoothly and have higher efficiency, though the trajectory of the joints could not be used directly to the robot because there was some difference on the size, freedoms and the actuator capability and so on between the animal and the mimetic robot.

\section{Motion planning}

According to the mechanism brought forward, a mode of gecko robot was built and exported to ADAMS for dynamic analysis of mechanical systems. The mode in ADAMS was showed in Fig. 6, by which the motion analysis after gait schemed was done.

It was found that during the experiments that gecko used trot gait (feet at the diagonal move nearly at the same time) most time in relative safe and certain circumstance, and used triangle gait (each foot lift off and drop down in turn, and three feet at stance phase and one 
at swing phase simultaneously) at times in some difficult situations, such as walking on the ceiling and so on. Of course it's not simultaneous exactly that each foot shifts in turn, but there exists transition time, in other words, four feet maybe at stance phase in a short time during transition. So in the motion planning we insert the transition state into the locomotion cycle.

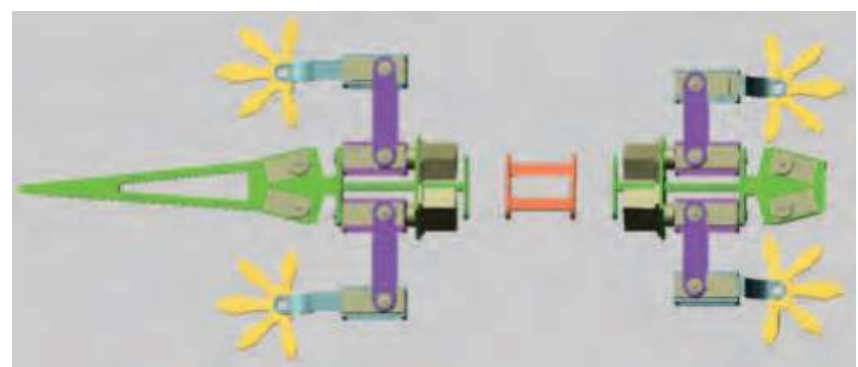

Fig. 6. ADAMS model of gecko-like robot IBSS-IV

\section{1 trot gait}

Just as diagram of trot gait showed in Fig. 7, the upper picture 0 was initial state. Picture 1, 3 was two motion states in which two legs in stance phase and the others two in swing phase. And picture 2, 4 was two transition states, in which two legs were in stance phase at the same time. The motion states 1, 2, 3 and 4, which correspond with the period in the below constitute the intact motion cycle.
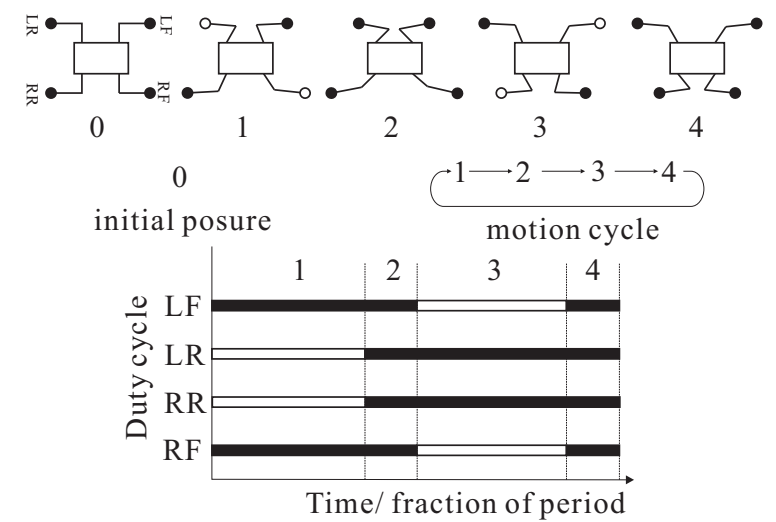

Fig. 7. Gait plan for trotting motion

Dynamic equilibrium exists in trot gait, and this is also complicated. ZMP (zero moment point) mode was a classic mode for the stability control and had been researched in depth(Kajita, 2007).

\subsection{Walk gait}

Triangle was often used when the circumstance is complex and at very low velocity. 
According to the result of gait characters of gecko, transition state that the four feet were all at stance phase inserted between the former and latter gait state. At one moment there's just one leg at swing phase, and other three legs at stance phase to drive the robot moving forward. The legs at left front, right rear, right front and left rear direction are at swing phase in turn. The sequence of each state was showed in Fig. 8, just as the motion of trot gait.

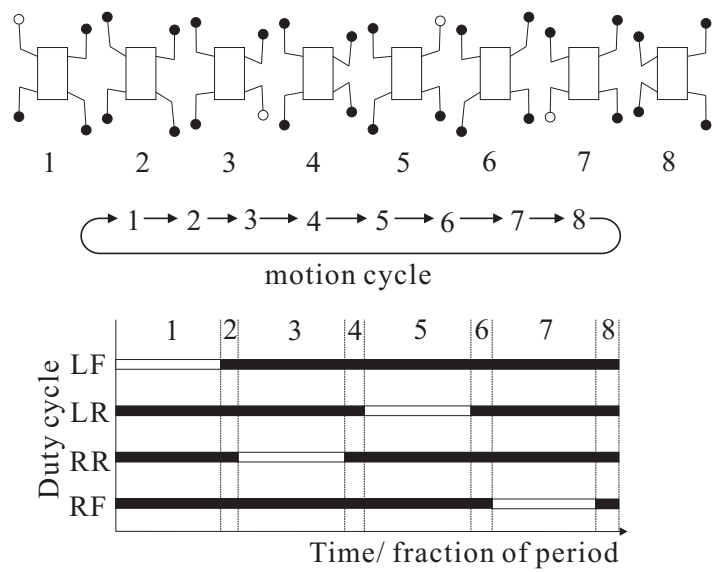

Fig. 8. Gait plan for walking motion

In Triangle gait static equilibrium, by which the center of gravity was kept in the triangle composed by the feet at stance phase, was often used in the control algorithm to keep the robot move stably. To avoid sliding between feet and contact surface, the relationship of friction angle and self-locking was widely used for the scheme of force applied to the feet. If the action line of active resultant force applied to the feet lying in the friction cone, sliding would be avoided no matter how much the active resultant force was because the selflocking had occurred. Inversely, if the action line of the resultant force was out of the friction cone, sliding must be occurred no matter how little the force was. And this principle was corresponding with the second and third situation in formula (1).

\section{Design of control system}

A distributed control system composed of one master - four slavers was designed to control the motion of robot. The master unit which was based on Atmega128L received orders from the host PC by wireless communication and distributed tasks to subsystems. Gait selection was also managed in the master. The subsystem was based on Atmega8L, which calculated the degrees each joints motor needed to drive the locomotion of robot leg. The I2C bus was applied to communicate between master and slaves, which need only 2 wires and the data rate up to $400 \mathrm{kHz}$. The whole control diagram was showed in Fig. 9. 


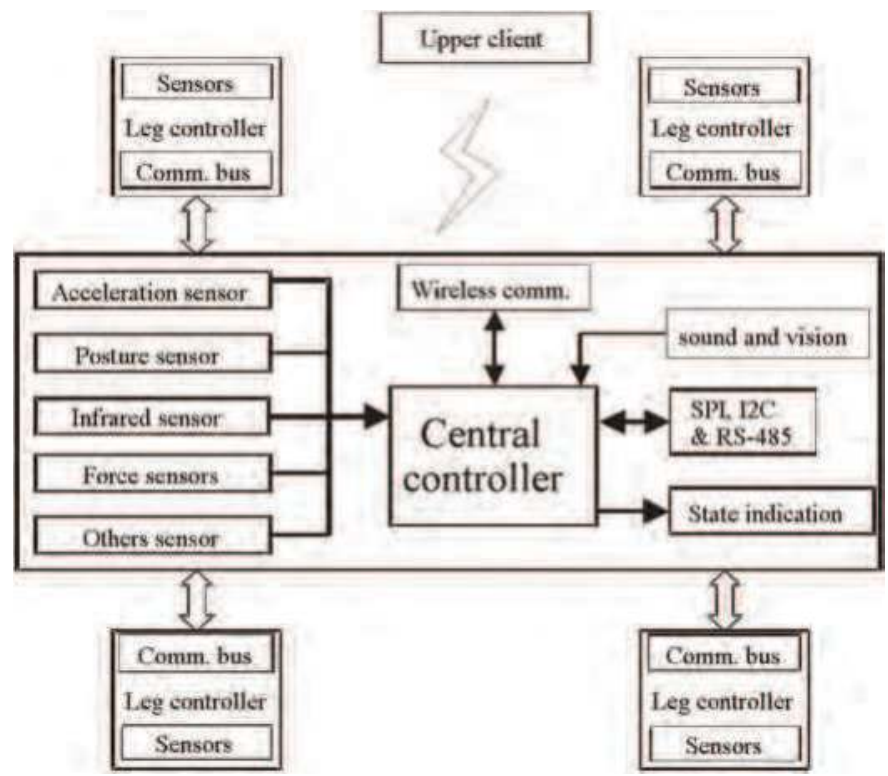

Fig. 9. Control diagram of gecko robot

Four foot pressure sensors were fixed on the sole to sense the state of feet. Foot state at each discrete step of all feet was sent to the master, according to the state master decided the next phase of each leg. The posture and acceleration sensor was used in the control system to sense the posture and motion state of robot during locomotion. Infrared sensor was for the distance detection and obstacle avoidance.

To improve the efficiency of the control system, the interrupt handing tasks were widely used on each mater and slaver unit, and the main program concentrated on the task receives, send and dispatch. And subsystem calculates the geometrical relation of each leg to control the rotation of each motor on each joint.

A high power Li-polymer battery was selected which could support high current. For the electrical reliability and stability of control system, the power of control and motors were separated. The real gecko robot was shown in Fig. 10.

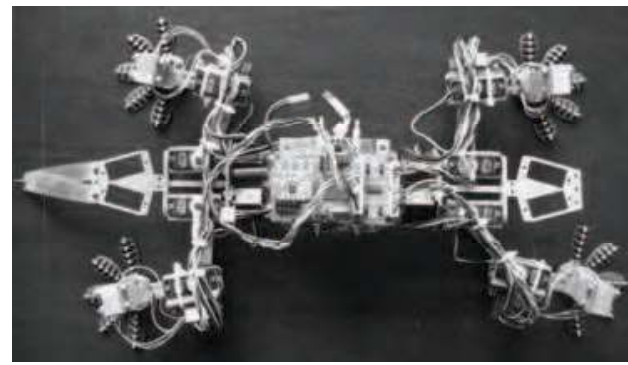

Fig. 10. A photo image of a gecko-like robot (IBSSIV) 


\section{Acknowledgement}

This subject was supported by the National High Technology Research and Development Program of China (Grant No. 2007AA04Z201) and the National Natural Science Foundation of China (Grant Nos. 60535020, 30700068 and 50705043).

\section{References}

Altendorfer, R. et al. (2001). RHex: a Biologically inspired hexapod runner. Autonomous Robots,Vol. 11, 207-213

Autumn, K. et al. (2006). Dynamics of geckos running vertically. Journal of Experimental Biology,Vol. 209, 260-272

Autumn, K. et al. (2000). Adhesive force of a single gecko foot-flair. Nature,Vol. 405, 681-685

Cartmill, M. (1985). Functional vertebrate morphology. Harvard University Press, Cambridge,MA

Collins, S. et al. (2005). Efficient bipedal robots based on passive-dynamic walkers. Science, Vol. 307, No.18, 1082-1085

Dai, J. S. \& Jones, J. R. (1999). Mobility in metamorphic mechanisms of foldable/erectable kinds. Journal of mechanical design,Vol. 121, No.3, 375-382

Dai et al. (2007). Motion mechanism bionics of discontinuous-Constraint Metamorphic robot: concept and model (in chinese). Chinese science bulletin, Vol. 52, No.2, 236-239

Damme, R. V.; Aerts, P. \& Vanhooybonck, B. (1998). Variation in morphology gait characteristics and speed of locomotion in two populations of lizards. Biological Journal of the Linnean Society,Vol. 63, 409-427

Damme, R. V.; Aerts, P. \& Vanhooydonck, B. (1997). No trade-off between sprinting and climbing in two populations of the Lizard Podarcis hispanica. Biological Journal of the Linnean Society, Vol. 60, 493-503

Dickinson, M. H. et al. (2000). How animals move: An Integrative view. Science,Vol. 288, No.7, 100-106

Eric, K. \& John, B. (1999). The Defense Advanced Research Projects Agency (DARPA) Tactical Mobile Robotics Program. Journal of Robotics Research,Vol. 18, No.7, 769-776

Jin, G.; Ding, X. \& Zhang, Q. (2004). Research on Configuration-complete Dynamics Modeling and Numerical Simulation of Metamorphic Mechanism(in Chinese). Acta Aeronautica ET Astronautica Sinica,Vol. 25, 401-405

Jin, G.; Gao, F. \& Ding, X. (2005). On Classification and Configuration Analysis for Metamorphic Mechanisms(in Chinese). Mechanical Science and Technology,Vol. 24, 64-767

Kajita, S. (2007). humanoid robot. Tsinghua University Press, 730214453, Beijing

Kim, S. et al. (2008). Smooth Vertical Surface Climbing With Directional Adhesion. IEEE transactions on robotics, Vol. 24, 65-74

Kristiaan, D. A. et al. (2002). Segment and Joint Angles of Hind Limb During Bipedal and Quadrupedal Walking of the Bonobo (Pan paniscus). American Journal of Physical Anthropology,Vol. 119, 37-51

Li, H. et al. (2009). Angular observation of joints of Geckos moving on horizontal and vertical surfaces. Chinese Science Bulletin,Vol. 54, No.4, 592-598

Liu, X. et al. (2005). A quantitative research on Gekko geckos appendicular muscle(in Chinese). Anatomy Research,Vol. 27, 292-301 
$\mathrm{Qu}$, L. et al. (2008). Biomimetic mushroom-shaped fibrillar adhesive microstructure Science,Vol. 322, No.238, 238-242

Raibert, M. et al. (2008). BigDog, the Rough-Terrain Quadruped Robot. Proceedings of Proceedings of the 17th World Congress the International Federation of Automatic Control,pp.10822-10825, 978-3-902661-00-5, Seoul, 2008,

Raibert, M. H. (1986). Legged robots that balance. MIT Press, Cambridge MA

Robert, G. et al. (2003). Small is beautiful: an army of small robots. Scientific American,Vol. 289, No.5, 63-66

Santos, D. et al. (2007). Directional adhesion for climbing: theoretical and practical considerations. Journal of Adhesion Science and Technology,Vol. 21, No.12, 1317-1341

Siegwart, R. \& Nourbakhsh, I. R. (2004). Introduction to Autonomous Mobile Robots. The MIT press, 0-262-19502-X, Massachusetts

Todd, D. J. (1985). Walking Machines: an Introduction to Legged Robots. Kogan Page Ltd, 085038-932-1, London

Wang, W. et al. (2008). A stereotaxic method and apparatus for the Gekko gecko. Chinese Science Bulletin,Vol. 53, No.7, 1107-1112

Winters, J. M. \& Crago, P. E. (2000). Biomechanics and neural control of posture and movement. Springer, New York

Zaaf, A. et al. (2001). Spatio-temporal gait characteristics of level and vertical locomotion in a ground-dwelling and a climbing gecko. The Journal of Experimental Biology,Vol. 204, 1233-1246

Zaaf, A. et al. (1999). Morphology and morphometrics of the appendicular musculature in geckoes with different locomotor habits (Lepidosauria). Zoomorphology,Vol. 119, 9-22 


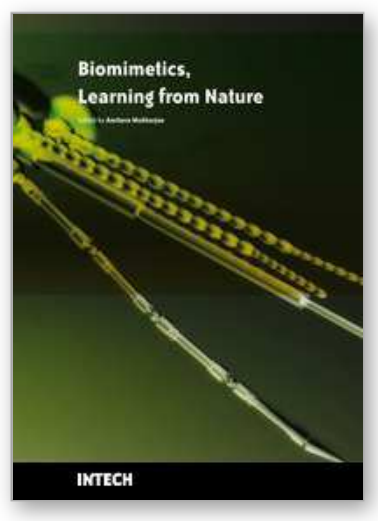

\section{Biomimetics Learning from Nature}

Edited by Amitava Mukherjee

ISBN 978-953-307-025-4

Hard cover, 534 pages

Publisher InTech

Published online 01, March, 2010

Published in print edition March, 2010

Nature's evolution has led to the introduction of highly efficient biological mechanisms. Imitating these mechanisms offers an enormous potential for the improvement of our day to day life. Ideally, by bio-inspiration we can get a better view of nature's capability while studying its models and adapting it for our benefit. This book takes us into the interesting world of biomimetics and describes various arenas where the technology is applied. The 25 chapters covered in this book disclose recent advances and new ideas in promoting the mechanism and applications of biomimetics.

\section{How to reference}

In order to correctly reference this scholarly work, feel free to copy and paste the following:

ZhenDong Dai and HongKai Li (2010). A Biomimetic Study of Discontinuous-Constraint Metamorphic Mechanism for Gecko-Like Robot, Biomimetics Learning from Nature, Amitava Mukherjee (Ed.), ISBN: 978953-307-025-4, InTech, Available from: http://www.intechopen.com/books/biomimetics-learning-from-nature/abiomimetic-study-of-discontinuous-constraint-metamorphic-mechanism-for-gecko-like-robot

\section{INTECH}

open science | open minds

\section{InTech Europe}

University Campus STeP Ri

Slavka Krautzeka 83/A

51000 Rijeka, Croatia

Phone: +385 (51) 770447

Fax: +385 (51) 686166

www.intechopen.com

\section{InTech China}

Unit 405, Office Block, Hotel Equatorial Shanghai

No.65, Yan An Road (West), Shanghai, 200040, China

中国上海市延安西路 65 号上海国际贵都大饭店办公楼 405 单元

Phone: +86-21-62489820

Fax: +86-21-62489821 
(C) 2010 The Author(s). Licensee IntechOpen. This chapter is distributed under the terms of the Creative Commons Attribution-NonCommercialShareAlike-3.0 License, which permits use, distribution and reproduction for non-commercial purposes, provided the original is properly cited and derivative works building on this content are distributed under the same license. 